\title{
Technique, protocols and adverse reactions for contrast-enhanced spectral mammography (CESM): a systematic review
}

Moreno Zanardo ${ }^{1}$, Andrea Cozzi ${ }^{1 *}$, Rubina Manuela Trimboli ${ }^{1}$, Olgerta Labaj ${ }^{2}$, Caterina Beatrice Monti ${ }^{1}$, Simone Schiaffino ${ }^{3}$, Luca Alessandro Carbonaro ${ }^{3}$ and Francesco Sardanelli ${ }^{1,3}$

\begin{abstract}
We reviewed technical parameters, acquisition protocols and adverse reactions (ARs) for contrast-enhanced spectral mammography (CESM). A systematic search in databases, including MEDLINE/EMBASE, was performed to extract publication year, country of origin, study design; patients; mammography unit/vendor, radiation dose, low-/highenergy tube voltage; contrast molecule, concentration and dose; injection modality, ARs and acquisition delay; order of views; examination time. Of 120 retrieved articles, 84 were included from 22 countries (September 2003January 2019), totalling 14012 patients. Design was prospective in 44/84 studies (52\%); in 70/84 articles (83\%), a General Electric unit with factory-set kVp was used. Per-view average glandular dose, reported in 12/84 studies (14\%), ranged $0.43-2.65 \mathrm{mGy}$. Contrast type/concentration was reported in 79/84 studies (94\%), with lohexol $350 \mathrm{mgl} / \mathrm{mL}$ mostly used $(25 / 79,32 \%)$, dose and flow rate in 72/84 (86\%), with $1.5 \mathrm{~mL} / \mathrm{kg}$ dose at $3 \mathrm{~mL} / \mathrm{s}$ in $62 / 72$ studies (86\%). Injection was described in 69/84 articles (82\%), automated in 59/69 (85\%), manual in 10/69 (15\%) and flush in 35/84 (42\%), with 10-30 mL dose in 19/35 (54\%). An examination time $<10$ min was reported in 65/84 studies (77\%), $120 \mathrm{~s}$ acquisition delay in 65/84 (77\%) and order of views in 42/84 (50\%) studies, beginning with the craniocaudal view of the non-suspected breast in $7 / 42$ (17\%). Thirty ARs were reported by $14 / 84$ (17\%) studies (26 mild, 3 moderate, 1 severe non-fatal) with a pooled rate of $0.82 \%$ (fixed-effect model). Only half of CESM studies were prospective; factory-set $\mathrm{kVp}$, contrast $1.5 \mathrm{~mL} / \mathrm{kg}$ at $3 \mathrm{~mL} / \mathrm{s}$ and $120 \mathrm{~s}$ acquisition delay were mostly used; only 1 severe AR was reported. CESM protocol standardisation is advisable.
\end{abstract}

Keywords: Breast, Contrast media, Drug-related side effects and adverse reactions, Mammography, Radiation dosage

\section{Key points}

- Eighty-four articles on CESM totalling 14012 patients were reviewed

- A $1.5 \mathrm{~mL} / \mathrm{kg}$ contrast dose automatically injected at $3 \mathrm{~mL} / \mathrm{s}$ was generally adopted

- Per-view average glandular dose ranged from 0.43 to 2.65 mGy

\footnotetext{
* Correspondence: andrea.cozzi1@unimi.it

'Department of Biomedical Sciences for Health, Università degli Studi di

Milano, Via Mangiagalli 31, 20133 Milan, Italy

Full list of author information is available at the end of the article
}

- Studies for contrast agent dose-finding and view acquisition ordering are lacking

- Adverse reaction rate (only one severe) was similar to that reported for CT

\section{Background}

During the 1960s and 1970s, randomised controlled trials proved that screen-film mammography for breast cancer screening yields a reduction in breast cancer mortality [1]. Since the early 2000s, screen-film mammography was progressively replaced by digital mammography (DM), which improved performance especially in women under 50 years of age and in case of 
dense breasts, even though providing an intrinsically inferior spatial resolution [2]. In the last two decades, digital breast tomosynthesis brought substantial further improvements $[3,4]$, increasing cancer detection rate and reducing the recall rate [5].

Contrast-enhanced mammography is the combination of X-ray mammography with intravenous administration of iodinated contrast agent (ICA) [6]. It was first attempted using a digital subtraction technique [7-9], but this approach was soon abandoned due to difficulties in co-registration of unenhanced and contrast-enhanced images $[10,11]$. In the last two decades, contrast-enhanced spectral mammography (CESM) has been introduced, based on dualenergy breast exposure (about $26-33 \mathrm{kVp}$ and 44$50 \mathrm{kVp}$ ) after contrast administration, so that the precontrast exposure was no longer needed [10, 12]. CESM allows for the visualisation of enhancing findings over the normal unenhancing breast tissue, exploiting the increased contrast uptake of malignancies $[6,10,13]$.

Original studies have investigated the use of CESM in a number of settings, such as evaluation of symptomatic women [14-17], screening recalls [18-22], local staging [23-32], pre- and post-operative evaluations [23, 24, 33-36] and neoadjuvant chemotherapy response monitoring [3740]. In 2016, a first meta-analysis on CESM described a high pooled sensitivity (98\%) albeit with a relatively low specificity (58\%) [41], the latter partly caused by inexperience. A more recent meta-analysis [42] reported globally satisfying data for CESM-pooled sensitivity (89\%) and specificity (84\%), proposing it as an alternative to contrast-enhanced magnetic resonance imaging (MRI) and even suggesting CESM as a "useful triage test for initial breast lesions assessment" [41].

A time delay between the first appearance of new imaging techniques and their implementation in diagnostic routine is expected for many reasons, including not only the definition of indications but also the reproducibility of results. The latter is strongly influenced by technique details, such as contrast agent concentration, dose and injection rate, breast compression and positioning, exposure parameters and acquisition protocol. Indeed, the fact that CESM is variably performed across different centres, without an agreed and standardised technique, does not come as a surprise: this circumstance echoes the one observed for contrast-enhanced breast MRI in the 1990s, now settled by the publication of detailed international guidelines [43-46].

Therefore, the aim of this work was to review CESM studies focusing on adopted technique, contrast agent issues and acquisition workflow. This effort is crucial for future CESM investigations to be reproducible and comparable.

\section{Methods}

\section{Study protocol}

No ethics committee approval was needed for this systematic review. The study protocol was registered on PROSPERO (protocol CRD42018118554), the international prospective register of systematic reviews [47]. This systematic review was reported according to the Preferred Reporting Items for Systematic Reviews and Meta-Analyses (PRISMA) statement [48].

\section{Search strategy and eligibility criteria}

In February 2019, a systematic search was performed on MEDLINE (PubMed, https://www.ncbi.nlm.nih.gov/ pubmed/), EMBASE (Elsevier), the Cochrane Library (Cochrane Database of Systematic Reviews) and the Cochrane Central Register of Controlled Trials for articles that reported or may have reported CESM technique. A controlled vocabulary (medical subject headings in PubMed and EMBASE thesaurus keywords in EMBASE) was used. The search string was (cesm OR 'contrast enhanced spectral mammography'/exp. OR 'dual energy mammography' OR 'contrast enhanced digital mammography'/exp. OR 'contrast-enhanced mammography' OR 'dual-energy subtraction mammography' OR cedm OR cedsm OR 'contrast enhanced spectral imaging' OR 'high energy and low energy digital mammography') AND ('procedures'/exp. OR 'method' OR 'methods' OR 'procedure' OR 'procedures' OR 'technique' OR 'acquisition'/exp. OR 'contrast medium'/exp. OR 'contrast agent' OR 'contrast dye' OR 'contrast material' OR 'contrast media' OR 'contrast medium' OR 'radiocontrast medium' OR 'radiography contrast medium' OR 'roentgen contrast medium' OR 'image processing'/exp. OR 'image processing' OR 'image processing, computer-assisted' OR 'processing, image').

The search was limited to original studies on humans published in English, French and Spanish on peerreviewed journals, with an available abstract. No publication date limits were applied. First article screening was performed by two independent readers (A.C. and M.Z., with 1- and 3-year experience in breast imaging, respectively) considering only title and abstract. Eligible articles were those that reported in the title or in the abstract the use of CESM technique or that could have contained these data in the manuscript. After downloading eligible articles, the full text was read for a complete assessment. Finally, references of included articles were handsearched to check for further eligible studies.

\section{Data extraction}

Data extraction was performed independently by the same two readers who performed the literature search. Disagreements were settled by consensus. For each analysed article, year of publication, institution (such as 
hospitals, imaging facilities, breast units including radiology sections or any other type of centre in which CESM is performed) and country origin as well as research groups, design, number of patients and demographics were retrieved. Mammography unit, vendor, radiation dose and technical features such as low- and high-energy peak kilovoltage $(\mathrm{kVp})$, anode/filter combinations and exposure parameters were also extracted. Moreover, contrast agent type, dose and concentration were retrieved, as well as injection modality, if manual or automated, flow rate and additional post-contrast saline flush or "bolus chaser" if present. Furthermore, mild, moderate or severe adverse reactions to ICAs were extracted alongside strategies for their prevention. Regarding the acquisition protocol, time between contrast injection and first image acquisition and maximum examination duration were extracted. Regarding the order of views, we reported the acquisition sequence of the standard mammographic projections considering the craniocaudal (CC) and the mediolateral oblique (MLO) views, including the first side acquired. Missing data were requested to authors.

\section{Evidence synthesis}

To avoid risk of data duplication bias, in case of articles published by the same research group, we considered the possibility of performing subgroup analysis: therefore, before delving into further analysis of protocol description, we chose to change our viewpoint from the number of articles reporting a specific protocol to the minimum number of times a protocol was reported by a single research group.

Regarding the pooled rate of adverse reactions related to ICA administration across studies, statistical analysis was performed using Comprehensive Meta-Analysis v2.2.057 (Biostat, Englewood, NJ, USA) using the metaanalysis model "Number of events and study population". $I^{2}$ statistics was first calculated to assess heterogeneity and the fixed-effect model was used to provide the rate of adverse reactions and $95 \%$ of confidence intervals (CI). The risk of publication bias was assessed by visually inspecting funnel plot and performing the Egger test [49].

\section{Results}

\section{Studies}

A flowchart of study selection is shown in Fig. 1. Of 120 retrieved articles, 84 (70\%), published between September 2003 and January 2019, were analysed [7-10, 13-40, 50-101]; 40/84 (48\%) being retrospective and 44/84 (52\%) prospective (43/44 monocentric $(98 \%)$ and $1 / 44$ multicentric (2\%); 54/84 (64\%) articles investigated CESM diagnostic performance, whereas 30/84 (36\%) focused on technical features. The geographic distribution of research groups is depicted in Fig. 2.

\section{Populations and settings}

Data synthesis is reported in Table 1 . The number of patients ranged from 5 [63] to 2303 [13], for a total of 14,012 patients, with mean or median age ranging from 45 years [40] to 66 years [23]. In 29/84 studies (35\%), CESM was performed on patients from comprehensive databases of heterogeneous settings, such as pre- or post-operative evaluation, adjuvant or neoadjuvant chemotherapy response monitoring and equivocal findings at conventional imaging. The remaining 55 studies $(65 \%)$ were individually centred on a unique setting. Twenty-seven studies (32\%) performed CESM on suspicious cases from conventional imaging and screening recalls, 11 studies (13\%) in a firstline screening setting, 7 (8\%) performed CESM exclusively for known cancer staging, 4 (5\%) in a pre-operative setting, $4(5 \%)$ to assess and monitor the response to adjuvant chemotherapy and $2(2 \%)$ in a post-operative setting.

Timing of CESM examination with menstrual cycle was reported only in $18 / 84$ studies (21\%). In 10/18 (56\%) articles, it was mentioned but not applied; in 6/18 (33\%), it was applied with a feasibility window between the 5 th and 14th day of menstrual cycle; in 2/18 (11\%), CESM was synchronously performed with MRI in different phases of menstrual cycle to evaluate and compare background parenchymal enhancement.

\section{Technical features and parameters}

In 70 out of 84 studies (83\%), different systems from General Electric Healthcare (Chicago, IL, USA) were used, all with a prototype or a commercial release of the SenoBright upgrade which is required to perform dualenergy contrast-enhanced imaging. Twelve out of 84 articles (14\%) reported the adoption of Selenia Dimensions Mammography Unit (Hologic Inc., Marlborough, MA, USA), while the remaining $2 / 84$ (3\%) studies were conducted with a Siemens Healthineers (Erlangen, Germany) Mammography System (Mammomat or Mammomat Inspiration).

The type of ICA used was not reported in five articles $[15,24,64,66,75]$, while in the remaining 79 studies (94\%), for a total of 13465 patients (96\%), six different molecules were used: Iohexol was the most frequently employed, being used in $42 / 79$ studies (53\%) for a total of $5049 / 13465$ patients (37\%), followed by Iopromide (18/79 studies, 23\%; 2798/13465 patients, $21 \%$ ), while Iobitridol, Iomeprol, Iopamidol and Ioversol were administered in the remaining studies (19/79 studies, 24\%; 5618/ 13465 patients, $42 \%)$. Iohexol was utilised at a concentration of $350 \mathrm{mg}$ iodine $/ \mathrm{mL}$ (25/42 studies, $60 \%$; 3330/5049 patients, $66 \%$ ) or $300 \mathrm{mg}$ iodine/mL (17/42 studies, $40 \%$; 1719/5049 patients, 34\%). Iopromide was also 


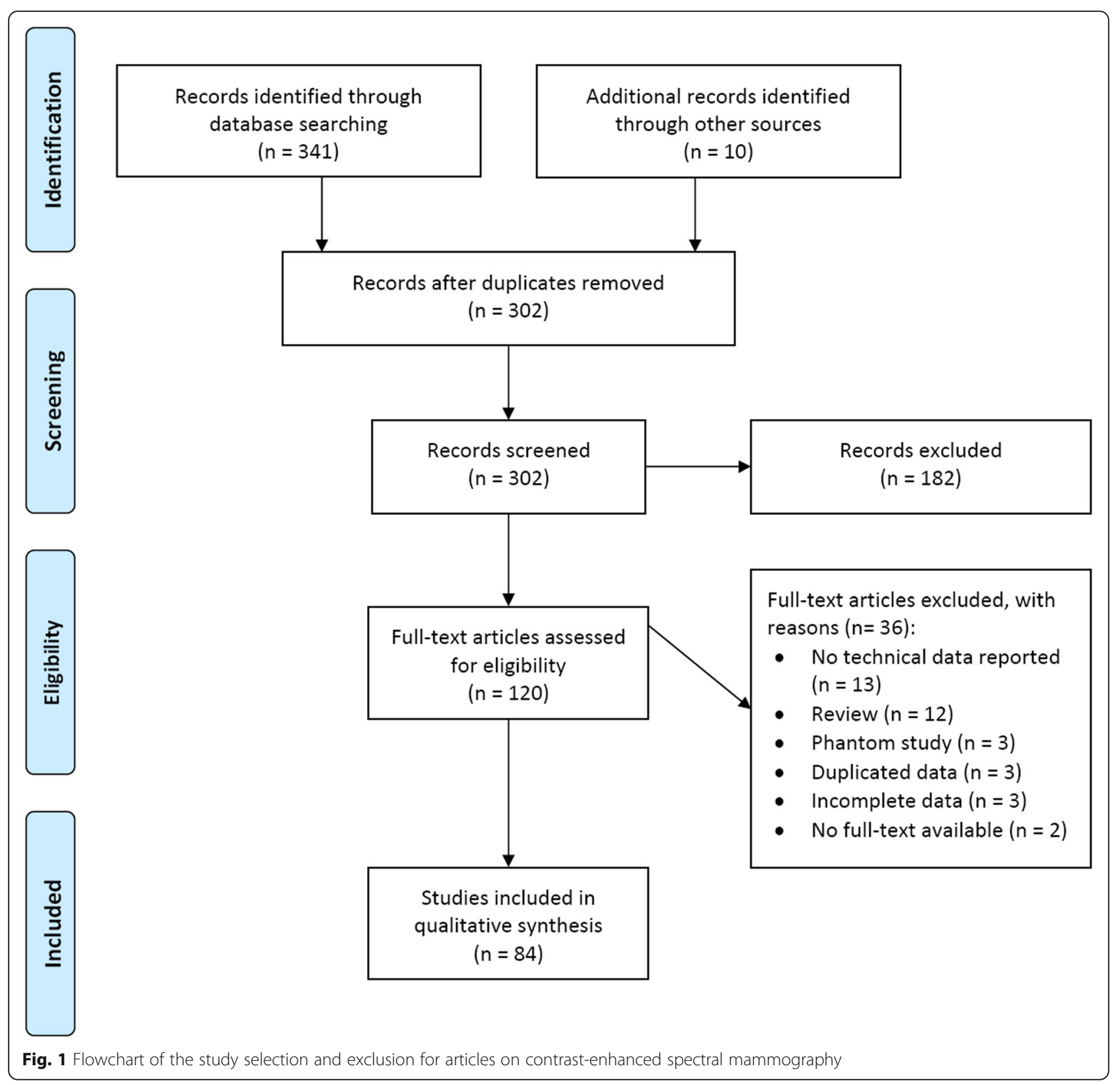

administered at two different concentrations: $370 \mathrm{mg}$ iodine/mL (10/18 studies, 56\%; 1032/2798 patients, 37\%) and $300 \mathrm{mg}$ iodine/mL (8/18 studies, $44 \% ; 1766 / 2798$ patients, $63 \%$ ).

Of the 69 studies including a specification of the contrast injection modality, 59 (85\%) utilised an automated power injector (10584/11725 patients, 90\%) while manual contrast injection was carried out in the remaining $10(15 \%)[7,9,17,25,28,51,57,73,95,99]$ for a total of $1141 / 11725$ patients (10\%).

Contrast agent dose, detailed in 77 studies, was fixed at $1.5 \mathrm{~mL} / \mathrm{kg}$ in $72(93 \%)$ of them for a total of $13559 / 13687$ (99\%) patients. Contrast agent flow rate, reported in 76/84 studies (90\%), was most frequently fixed at $3 \mathrm{~mL} / \mathrm{s}(65 / 76$ studies, $86 \%$ ); the 11 remaining articles detailed a flow rate ranging from 2 to $5 \mathrm{~mL} / \mathrm{s}$. Thirty-five out of 84 (42\%) articles for a total $8734 / 14012$ patients (62\%) also mentioned the use of additional post-contrast saline flush or "bolus chaser," 19 of them (54\%, for a total $4477 / 8734$ patients, $51 \%$ ) likewise detailing a saline amount ranging from 10 to $30 \mathrm{~mL}$.

Of 69 studies detailing the tube voltage of both low- and high-energy acquisitions, all but one (99\%) acquired lowenergy images between 26 and $33.2 \mathrm{kVp}$, which is the peak kilovoltage threshold of iodine, while all 69 acquired highenergy images well above this threshold, i.e. between 44 and $50 \mathrm{kVp}$. The anode/filter combination was reported 


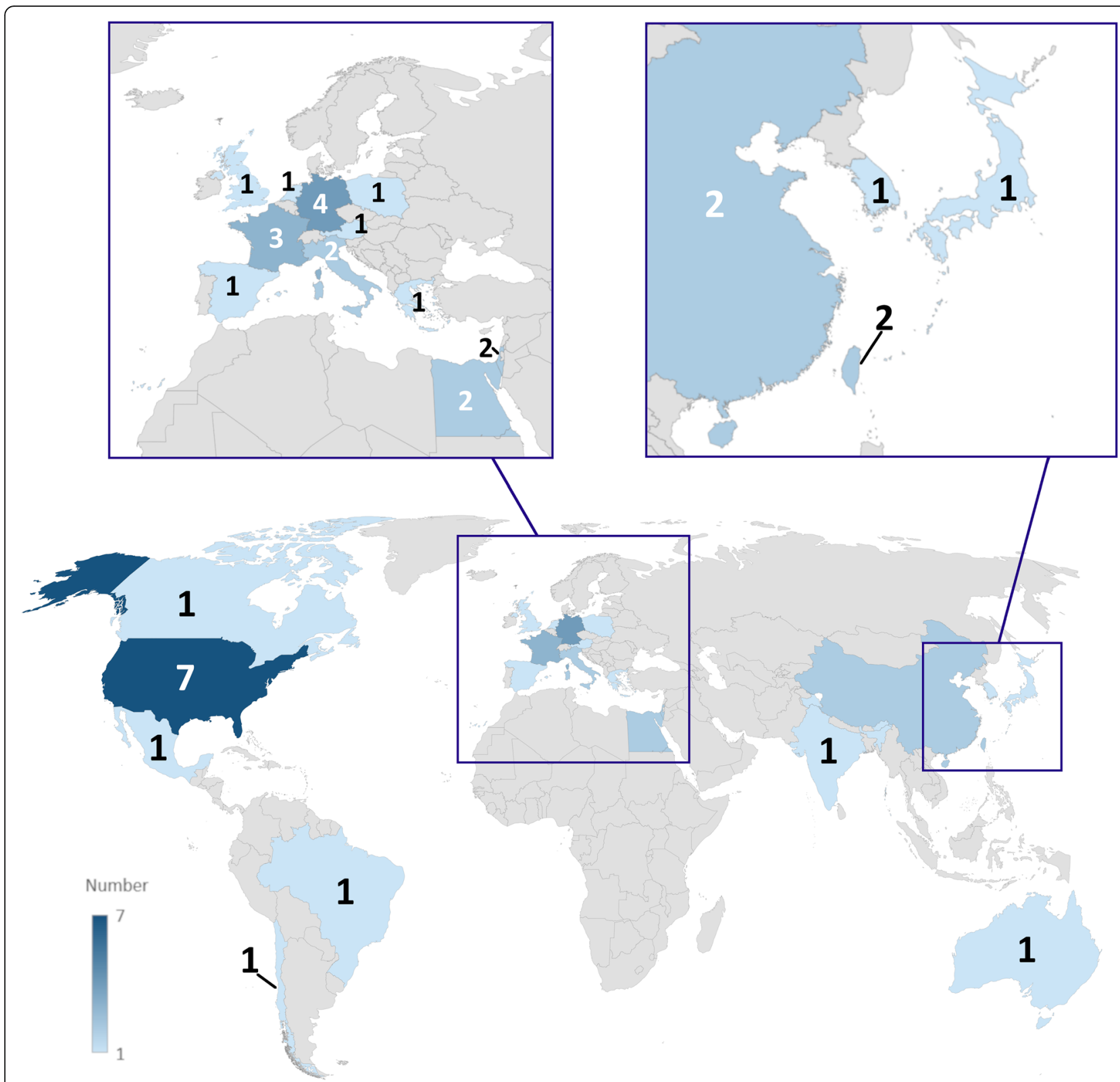

Fig. 2 Geographic distribution of research groups which published results of clinical applications of contrast-enhanced spectral mammography. From very light blue to dark blue, the number of groups progressively increases from 1 to 7 ; grey colour means no publications

by $42 / 84$ studies. Exposure parameters were unambiguously reported only in one study [10], whereas in 5 early studies $[7,8,32,59,85]$, they were manually adjusted according to breast thickness and density; thirty-five other studies declared an automatic regulation of these parameters performed by the mammography unit.

Regarding radiation dose, data were scarcer: even though 45/84 articles (54\%) mentioned this aspect, 17/ $45(31 \%)$ did it without exhibiting original information but reporting observations from previous studies, therefore restricting the number of studies with new data to 28/84 (33\%). Of these 28 studies, 19 (68\%) provided an average glandular dose (AGD), 3 (16\%) of them calculating it per-patient and ranging $1.5-6.9 \mathrm{mGy}[8,9,58], 5 /$ 19 (26\%) calculating it per-breast ranging 2.19-7.15 mGy and the remaining $11(58 \%)$ reporting a per-view AGD ranging from 0.43 [61] to $2.65 \mathrm{mGy}$ [101]. A comparison with DM was mentioned in 17 studies: only $1(6 \%)$ documented a dose reduction $(-2 \%)$ for CESM compared to DM [32], while other 16 (94\%) reported an increase in AGD ranging between 6.2\% [85] and 100\% [77]. However, it is worth to notice that 3 studies specifically contrived to assess CESM radiation doses reported an AGD increase of $42 \%$ [56], 78\% [82] and 80\% [60]. 
Table 1 Main characteristics of the 84 analysed studies

\begin{tabular}{|c|c|c|c|c|c|c|c|c|c|c|c|}
\hline Author/year & Ref. & $\begin{array}{l}\text { Study } \\
\text { design }\end{array}$ & $\begin{array}{l}\text { Country of } \\
\text { research group }\end{array}$ & $\begin{array}{l}\text { Number } \\
\text { of patients }\end{array}$ & $\begin{array}{l}\text { Mean or } \\
\text { median age } \\
\text { (years) }\end{array}$ & $\begin{array}{l}\text { Contrast } \\
\text { agent type }\end{array}$ & $\begin{array}{l}\text { Concentration } \\
(\mathrm{mgl} / \mathrm{mL})\end{array}$ & $\begin{array}{l}\text { Dose } \\
(\mathrm{mL} / \mathrm{kg})\end{array}$ & $\begin{array}{l}\text { Flow } \\
\text { rate } \\
(\mathrm{mL} / \mathrm{s})\end{array}$ & $\begin{array}{l}\text { Delay after } \\
\text { injection (s) }\end{array}$ & $\begin{array}{l}\text { Total } \\
\text { exam } \\
\text { time }\end{array}$ \\
\hline Houben 2019 & {$[22]$} & $\mathrm{R}$ & The Netherlands & 147 & 61 & lopromide & 300 & 1.5 & 3 & 120 & \\
\hline Barra 2018 & [40] & P mono & Brazil & 33 & 45 & lohexol & 300 & 1.5 & 3 & 120 & B \\
\hline Bicchierai 2018 & [93] & $\mathrm{R}$ & Italy & 40 & 50 & lopromide & 370 & 1.5 & 3 & 120 & B \\
\hline Danala 2018 & [69] & $\mathrm{R}$ & USA & 111 & & lohexol & 350 & 1.5 & 3 & 120 & B \\
\hline Deng 2018 & [78] & $\mathrm{R}$ & Taiwan & 141 & 48 & lohexol & 350 & 1.5 & 3 & 120 & B \\
\hline Helal 2018 & [25] & P mono & Egypt & 300 & 54 & lohexol & 300 & 1.5 & 3 & 120 & B \\
\hline Kim 2018 & [87] & P mono & South Korea & 84 & 51 & lohexol & 350 & 1.5 & 2 & 120 & B \\
\hline Klang 2018 & [88] & $\mathrm{R}$ & Israel & 953 & 51 & lopamidol & 370 & 1.5 & 3 & 120 & B \\
\hline Łuczyńska 2018 & {$[36]$} & $\mathrm{R}$ & Poland & 82 & 57 & lopromide & 370 & 1.5 & 3 & 120 & B \\
\hline Moustafa 2018 & [17] & P mono & Egypt & 160 & & lohexol & 300 & 1.5 & 3 & 120 & B \\
\hline Navarro 2018 & [90] & P mono & Chile & 465 & 53 & loversol & 320 & 1.5 & & & B \\
\hline Patel 2018 (01) & [38] & P mono & USA & 65 & 53 & lohexol & 350 & 1.5 & 3 & 120 & A \\
\hline Patel 2018 (02) & [34] & $\mathrm{R}$ & USA & 50 & 57 & lohexol & 350 & 1.5 & 3 & 120 & B \\
\hline Patel 2018 (03) & [23] & $\mathrm{R}$ & USA & 30 & 66 & lohexol & 350 & 1.5 & 3 & 120 & B \\
\hline Phillips 2018 & [82] & $\mathrm{R}$ & USA & 45 & 53 & lohexol & 350 & 1.5 & 3 & 120 & \\
\hline Sorin 2018 & [92] & $\mathrm{R}$ & Israel & 611 & 54 & lopamidol & 370 & 1.5 & 3 & 120 & B \\
\hline Tohamey 2018 & [51] & P mono & Egypt & 178 & 46 & lohexol & 300 & 1.5 & 3 & 120 & B \\
\hline Travieso-Aja 2018 & [24] & $\mathrm{R}$ & Spain & 158 & 51 & & & 1.5 & 3 & 120 & B \\
\hline Xing 2018 & [84] & P mono & China & 235 & 51 & lohexol & 350 & 1.5 & 3 & 120 & B \\
\hline Barra 2017 & [39] & $\mathrm{R}$ & Brazil & 11 & 46 & lohexol & 300 & $1-2$ & 3 & 120 & B \\
\hline Bhimani 2017 & [13] & $\mathrm{R}$ & USA & 2303 & & lopamidol & 370 & 1.5 & 2 & 120 & B \\
\hline Fallenberg 2017 & [76] & P multi & Germany & 155 & 53 & lobitridol & 300 & 1.5 & 3 & 120 & A \\
\hline Gluskin 2017 & [63] & $\mathrm{R}$ & USA & 5 & 59 & lohexol & 350 & 1.5 & 3 & 150-180 & A \\
\hline Helal 2017 (01) & {$[28]$} & P mono & Egypt & 98 & 50 & lohexol & 300 & 1.5 & 3 & 120 & B \\
\hline Helal 2017 (02) & [99] & P mono & Egypt & 30 & 47 & lohexol & 300 & 1.5 & & 120 & \\
\hline Houben 2017 & [58] & $\mathrm{R}$ & The Netherlands & 839 & 60 & lopromide & 300 & 1.5 & 3 & 120 & \\
\hline lotti 2017 & [37] & P mono & Italy & 54 & 54 & loversol & 350 & 1.5 & & 120 & \\
\hline James 2017 & {$[56]$} & $\mathrm{R}$ & USA & 173 & & lohexol & 350 & 1.5 & 3 & 120 & A \\
\hline Jochelson 2017 & [54] & P mono & USA & 309 & 51 & lohexol & 350 & 1.5 & 3 & $150-180$ & B \\
\hline Knogler 2017 & [94] & P mono & Austria & 11 & 58 & lomeprol & 400 & 2 & 3.5 & 90 & \\
\hline Lee-Felker 2017 & {$[26]$} & $\mathrm{R}$ & USA & 52 & 50 & lohexol & 350 & & 3 & 120 & B \\
\hline Lewis 2017 & [16] & $\mathrm{R}$ & USA & 208 & & lohexol & 350 & 1.5 & 3 & 120 & B \\
\hline Li 2017 & {$[100]$} & $\mathrm{R}$ & USA & 48 & 56 & lopamidol & 370 & 1.5 & $1.5-2$ & & B \\
\hline Mori 2017 & [74] & P mono & Japan & 72 & 48 & lohexol & 300 & 1.5 & 3 & 120 & \\
\hline Patel 2017 (01) & [27] & $\mathrm{R}$ & USA & 88 & 62 & lohexol & 350 & 1.5 & 3 & 120 & B \\
\hline Patel 2017 (02) & {$[65]$} & $\mathrm{R}$ & USA & 410 & & lohexol & 350 & 1.5 & 3 & 120 & B \\
\hline Phillips 2017 & [70] & P mono & USA & 38 & 53 & lohexol & 350 & 1.5 & 3 & 120 & B \\
\hline Richter 2017 & {$[62]$} & $\mathrm{R}$ & Germany & 118 & 58 & lopromide & 300 & 1.5 & $2-3$ & 120 & \\
\hline Saraya 2017 & [18] & P mono & Egypt & 34 & 54 & lohexol & 300 & 1.5 & 4 & & C \\
\hline Savaridas 2017 & {$[75]$} & P mono & Australia & 66 & 54 & & & 1.5 & 3 & 120 & B \\
\hline Sogani 2017 & [80] & $\mathrm{R}$ & USA & 278 & 51 & lohexol & 350 & 1.5 & 3 & 150 & A \\
\hline Ali-Mucheru 2016 & [33] & $\mathrm{R}$ & USA & 351 & 62 & lohexol & 350 & 1.5 & 3 & 120 & B \\
\hline Ambicka 2016 & [29] & $\mathrm{R}$ & Poland & 82 & 57 & lopromide & 370 & 1.5 & 3 & 120 & B \\
\hline
\end{tabular}


Table 1 Main characteristics of the 84 analysed studies (Continued)

\begin{tabular}{|c|c|c|c|c|c|c|c|c|c|c|c|}
\hline Author/year & Ref. & $\begin{array}{l}\text { Study } \\
\text { design }\end{array}$ & $\begin{array}{l}\text { Country of } \\
\text { research group }\end{array}$ & $\begin{array}{l}\text { Number } \\
\text { of patients }\end{array}$ & $\begin{array}{l}\text { Mean or } \\
\text { median age } \\
\text { (years) }\end{array}$ & $\begin{array}{l}\text { Contrast } \\
\text { agent type }\end{array}$ & $\begin{array}{l}\text { Concentration } \\
(\mathrm{mgl} / \mathrm{mL})\end{array}$ & $\begin{array}{l}\text { Dose } \\
(\mathrm{mL} / \mathrm{kg})\end{array}$ & $\begin{array}{l}\text { Flow } \\
\text { rate } \\
(\mathrm{mL} / \mathrm{s})\end{array}$ & $\begin{array}{l}\text { Delay after } \\
\text { injection (s) }\end{array}$ & $\begin{array}{l}\text { Total } \\
\text { exam } \\
\text { time }\end{array}$ \\
\hline Brandan 2016 & [77] & P mono & Mexico & 18 & 51 & loversol & 300 & & 4 & 60 & B \\
\hline Cheung 2016 (01) & [72] & $\mathrm{R}$ & Taiwan & 256 & 48 & lohexol & 350 & 1.5 & 3 & 120 & A \\
\hline Cheung 2016 (02) & [98] & $\mathrm{R}$ & Taiwan & 87 & 54 & lohexol & 350 & 1.5 & 3 & 120 & B \\
\hline Kamal 2016 & [95] & $\mathrm{R}$ & Egypt & 239 & 48 & lohexol & 300 & 1.5 & 3 & 120 & B \\
\hline Kariyappa 2016 & [68] & P mono & India & 44 & & lomeprol & 350 & 1.5 & 3 & 120 & B \\
\hline Knogler 2016 & [83] & P mono & Austria & 15 & 58 & lomeprol & 400 & 2 & 3.5 & $60-90$ & \\
\hline Lalji 2016 & [21] & $\mathrm{R}$ & The Netherlands & 199 & 58 & lopromide & 300 & 1.5 & 3 & 120 & \\
\hline Łuczyńska 2016 (01) & [50] & P mono & Poland & 116 & 55 & lopromide & 370 & 1.5 & 3 & 120 & B \\
\hline Łuczyńska 2016 (02) & [67] & P mono & Poland & 193 & 55 & lopromide & 370 & 1.5 & 3 & 120 & B \\
\hline Tardivel 2016 & [19] & R & France & 195 & 56 & lobitridol & 300 & 1.5 & 3 & 120 & B \\
\hline Tennant 2016 & [15] & $\mathrm{R}$ & UK & 99 & 49 & & & & & & \\
\hline Tsigginou 2016 & [89] & P mono & Greece & 216 & 55 & lopromide & 300 & 1.5 & $2-3$ & 120 & B \\
\hline Wang 2016 & [97] & P mono & China & 68 & 53 & lohexol & 350 & 1.5 & 3 & 120 & A \\
\hline Yagil 2016 & [71] & R & Israel & 200 & 51 & lopamidol & 370 & 1.5 & 3 & 120 & B \\
\hline Chou 2015 & [14] & P mono & Taiwan & 185 & 51 & lohexol & 300 & 1.5 & 2 & 120 & B \\
\hline Elsaid 2015 & [73] & P mono & Egypt & 34 & 55 & lohexol & 300 & 1.5 & 3 & & B \\
\hline Hobbs 2015 & [81] & P mono & Australia & 49 & 55 & lohexol & 350 & 1.5 & 3 & 120 & B \\
\hline Kamal 2015 & [79] & $\mathrm{R}$ & Egypt & 168 & & lohexol & 300 & 1.5 & 3 & 120 & B \\
\hline Lobbes 2015 & [30] & R & The Netherlands & 87 & 62 & lopromide & 300 & 1.5 & 3 & 120 & \\
\hline Łuczyńska 2015 (01) & [91] & P mono & Poland & 174 & 56 & lopromide & 370 & 1.5 & 3 & 120 & B \\
\hline Łuczyńska 2015 (02) & [53] & P mono & Poland & 102 & & lopromide & 370 & 1.5 & 3 & 120 & \\
\hline Badr 2014 & [101] & P mono & France & 75 & 54 & lohexol & 300 & 1.5 & & 120 & B \\
\hline Blum 2014 & [31] & P mono & Germany & 20 & 57 & lopamidol & 300 & 1.5 & 3 & 120 & \\
\hline Cheung 2014 & [86] & R & Taiwan & 89 & 48 & lohexol & 350 & 1.5 & 3 & $120-180$ & B \\
\hline Fallenberg 2014 (01) & [85] & P mono & Germany & 118 & 53 & lobitridol & 300 & 1.5 & 3 & 120 & B \\
\hline Fallenberg 2014 (02) & [32] & P mono & Germany & 80 & 54 & lobitridol & 300 & 1.5 & 3 & 120 & B \\
\hline Francescone 2014 & [66] & $\mathrm{R}$ & USA & 88 & 50 & & & & & & \\
\hline Jeukens 2014 & [60] & $\mathrm{R}$ & The Netherlands & 47 & 58 & lopromide & 300 & 1.5 & 3 & 120 & \\
\hline Lobbes 2014 & [20] & R & The Netherlands & 113 & 57 & lopromide & 300 & 1.5 & 3 & 120 & \\
\hline Łuczyńska 2014 & [35] & P mono & Poland & 152 & 56 & lopromide & 370 & 1.5 & 3 & 120 & B \\
\hline Mokhtar 2014 & [57] & P mono & Egypt & 60 & & lohexol & 300 & 1.5 & & 120 & A \\
\hline Travieso-Aja 2014 & [64] & $\mathrm{R}$ & Spain & 136 & 49 & & & 1.5 & 3 & 120 & B \\
\hline Hill 2013 & [10] & $R$ & Canada & 98 & 57 & lobitridol & 300 & 1.5 & 3 & 120 & B \\
\hline Jochelson 2013 & [55] & P mono & USA & 82 & 50 & lohexol & 350 & 1.5 & 3 & $150-300$ & B \\
\hline Dromain 2012 & [52] & P mono & France & 110 & 57 & lobitridol & 300 & 1.5 & 3 & 120 & A \\
\hline Diekmann 2011 & [61] & P mono & Germany & 70 & 55 & lopromide & 370 & 1 & 4 & $60 / 120 / 180$ & A \\
\hline Dromain 2011 & [59] & P mono & France & 120 & 56 & lobitridol & 300 & 1.5 & 3 & 120 & A \\
\hline Dromain 2006 & [9] & P mono & France & 20 & 63 & lohexol & 300 & & 3 & 30 & B \\
\hline Diekmann 2005 & [8] & P mono & Germany & 21 & & lopromide & 370 & 1 & 4 & $60 / 120 / 180$ & A \\
\hline Jong 2003 & [7] & P mono & Canada & 22 & & lohexol & 300 & & & 60 & B \\
\hline Lewin 2003 & [96] & P mono & USA & 26 & 51 & lohexol & 350 & & $4-5$ & 150 & \\
\hline
\end{tabular}

$R$ retrospective, $P$ mono prospective monocentric, $P$ multi prospective multicentric, $A=$ total exam time $<5$ min, $B=$ total exam time between 5 and 10 min, $C=$ total exam time $>10 \mathrm{~min}$ 


\section{Acquisition protocols}

Studies reporting the time interval between contrast injection and the first image acquisition were 78 out of 84 (93\%), for a total $13244 / 14012$ patients (95\%) and $65(83 \%)$ of them (12278/13244 patients, 93\%) had it fixed at $120 \mathrm{~s}$.

Sixty-six out of 84 articles (79\%, 11900/14012 patients, $85 \%)$ gave an indication of the acquisition time after contrast injection: in $12 / 66$ (18\%, 1381/11900 patients, $11.6 \%)$, the exam was completed in less than $5 \mathrm{~min}$; in $52 / 66(80 \%$, for total of $10485 / 11900$ patients, $88.1 \%$ ) between 5 and 10 min, while in $1 / 66(2 \%, 34 / 11900$ patients, $0.3 \%)$ the duration exceeded $10 \mathrm{~min}$.

The outline of the image acquisition sequence remains more variable. Ten out of 84 studies (12\%), accounting for 2734 patients $(19 \%)$ did not clearly describe it and did not provide a reference to other protocols, while $3 / 84(4 \%$, 103/14012 patients, 1\%) employed a curtailed and sideinsensitive acquisition sequence. Adherence to standard but unspecified digital mammography protocols was declared by $29 / 84$ (34\%) studies, for total $3741 / 14012$ patients $(27 \%)$. The other half of the articles analysed (42/84, accounting for 7434/14012 patients, 53\%) unequivocally detailed an acquisition sequence. Of these 42 studies, 14 (34\%, 2048/7434 patients, 28\%) adopted a projection order that was conventionally agreed upon, while the other 28 (66\%, accounting for 5386/7434 patients, $72 \%$ ) based their acquisition sequence on the presence of previous suspect or clearly pathologic findings.

Eighty-four articles came from 38 different research groups. Subgroup analysis according to research groups showed that 17 acquisition sequences based on a conventionally agreed projection order were executed in 15 research groups. As described in Fig. 3, the most common sequence description, reported by $6 / 17$ (35\%) institutions, was MLO - MLO - CC - CC (in order of acquisition), without any further indication about the first side to be examined (right or left or side with/without suspicious lesion or already diagnosed cancer). The second most common sequence $(4 / 17,24 \%)$ was CC - CC - MLO - MLO with the first projection standardised on the right side (independently of pathology or with suspected pathology).

Among the 22 acquisition sequences (coming from 20 institutions) centred on the presence of previous suspect or clearly pathologic findings, we found substantial variability between different orders of acquisition, as shown in Fig. 4. However, the most common sequence, adopted by $4 / 22(19 \%)$ research groups, was 1$)$ CC, suspected side; 2) CC, non-suspected side; 3) MLO, suspected side; and 4) MLO, non-suspected side.

\section{Contrast agent adverse reaction rate meta-analysis}

Regarding side effects from ICA administration, 48/84 studies (57\%) declared a preventive anamnestic screening for previous adverse reactions or general contraindications to ICA administration. Pre-examination tests of renal function was mentioned in $39 / 84$ studies (46\%). Of note, $14 / 84$ studies (29\%) reported 30 adverse reactions out of 14012 patients, of which $26 / 30$ (87\%) were mild reactions limited to pruritus, hives, "scratchy throat" or other minor skin flushing that resolved promptly even when antihistamines or corticosteroids were not administered. In 3/30 (10\%) cases [54, $58,87]$, side effects were of moderate importance with nausea and vomiting, widespread urticaria resolved only after antihistamines and corticosteroids per os, and dyspnea that equally responded to oral antihistamine administration. Only $1 / 30$ (3\%) severe adverse reaction, requiring "intensive care" but resolved after short time, occurred in 14012 patients $(0.007 \%)$ [61].

Therefore, the number of adverse reactions related to ICA administration ranged from 0 , reported by $70(88 \%)$ studies, to a maximum of 6 adverse reactions [14] with a total of 30 adverse reactions, showing no heterogeneity $\left(Q=64\right.$, degree of freedom 83, $\tau=2.0972, I^{2}=0 \%, p=$ 0.931). As shown in the forest plot of Fig. 5, using fixedeffect model, the pooled rate of adverse reactions across studies was $0.82 \%$, with $0.64 \%$ and $1.05 \%$ as $95 \%$ CI.

Visually inspecting the funnel plot in Fig. 6, risk of publication bias was found, as confirmed by the Egger test $(p=0.00028)$.

\section{Discussion}

Our systematic review included 84 articles, accounting for 14012 patients, reporting the use of CESM in various settings. The sheer number of studies and, as depicted in Fig. 7, their increase in the last 3 years $(27$ studies between 2003 and December 2015, 57 from January 2016 to January 2019) points out a considerable interest in this emerging breast imaging modality.

A number of narrative reviews [6, 42, 102-106] favourably outlined CESM future perspectives in several clinical settings (e.g. recall work-up, pre-operative staging, and monitoring the effect of neoadjuvant therapy) as a potential alternative to MRI.

In the first phase of CESM development, some nonfixed parameters regarding contrast agent administration (i.e. contrast agent molecule, concentration, dose, flow rate, and injection modality) and some acquisition features (i.e. time between contrast injection and first acquisition, $\mathrm{kVp}$ ranges for low- and high-energy acquisitions) gained an international agreement. However, in the framework of comprehensive optimisation and standardisation of CESM, large-scale studies are undoubtedly needed to address the knowledge gap concerning the choice of technical parameters.

Our data show a consensus among studies (93\%) on the choice of $1.5 \mathrm{~mL} / \mathrm{kg}$ contrast dose administered with a 3 $\mathrm{mL} / \mathrm{s}$ flow rate $(74 \%)$ and a less extensive agreement on the 


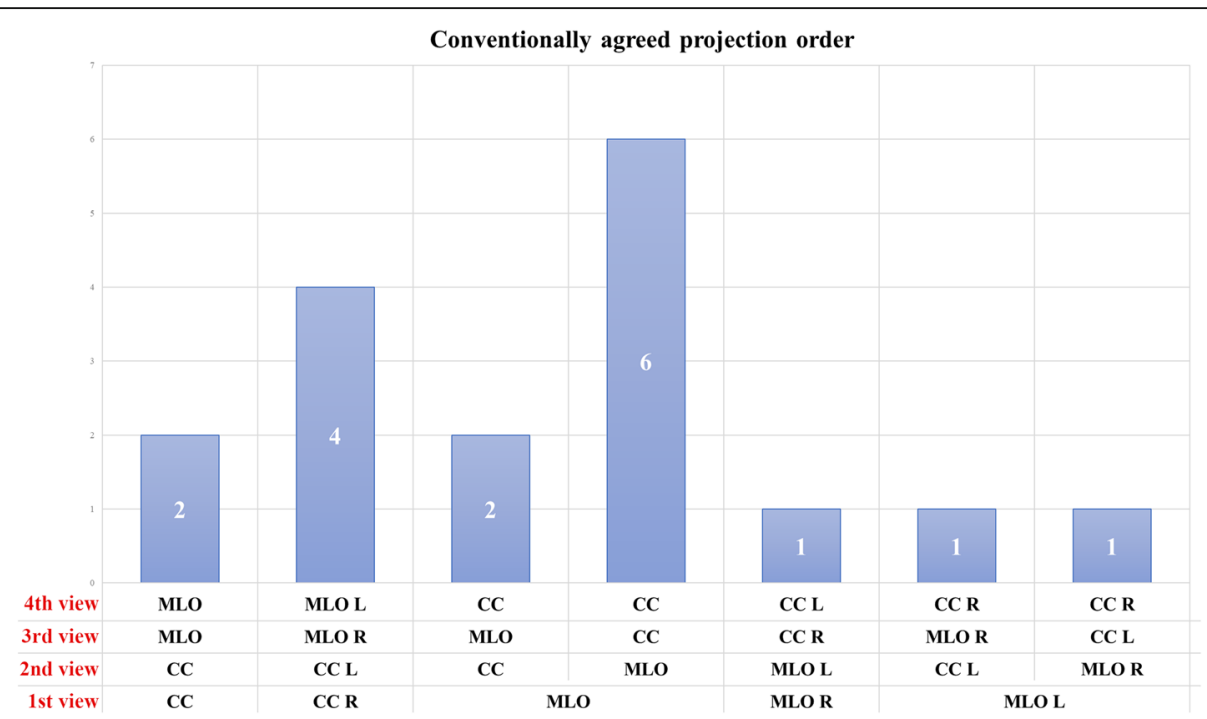

Fig. 3 Graphical summary of conventionally agreed view acquisition orders for contrast-enhanced spectral mammography: CC craniocaudal view, MLO mediolateral oblique view, $L$ left, $R$ right

use of Iohexol (53\% of all studies) at a concentration of 350 $\mathrm{mg}$ iodine $/ \mathrm{mL}$ (30\% of all studies). However, these parameters have probably been empirically adopted from CT protocols, as the first investigators plainly stated [7], without any other particular explication or justification. No dose-finding studies have been published yet.

Similarly, the common use of a power contrast injector (87\% of all studies, with the remaining $13 \%$ coming from a single research group) is assumed from CT and MRI protocols in which it has been demonstrated to be effective in obtaining a stable contrast inflow and bolus shape [107109]. Moreover, the use of a power injector allows for the administration of a bolus chaser, reported only in $42 \%$ of all articles, a technical refinement that has shown good results in CT [110, 111].

Two other points need to be mentioned. The first one is the correlation between menstrual cycle phase and background parenchymal enhancement, explored in a few studies $[10,75,80]$ and/or fluctuations of lesion contrast uptake. Secondly, since CESM is based on a dual $\mathrm{X}$-ray exposure, of which the low-energy one has been demonstrated to be equal to standard DM [66], an increase in radiation dose is expected. However, while preliminary studies estimated a negligible [7] or curtailed

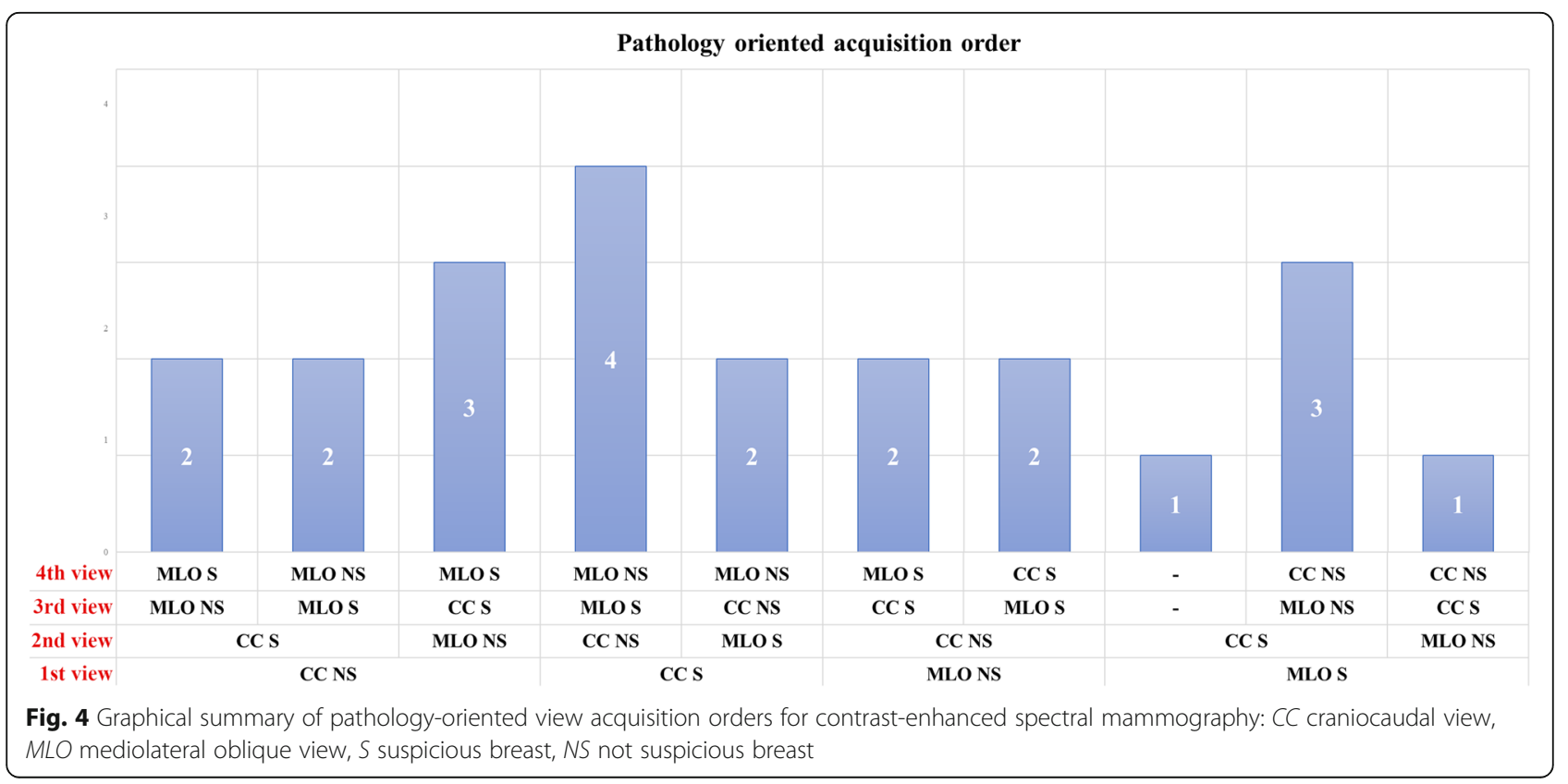




\begin{tabular}{|c|c|c|c|c|c|c|c|}
\hline \multirow[t]{2}{*}{ Study name } & \multicolumn{5}{|c|}{ Statistics for each study } & & \\
\hline & $\begin{array}{c}\text { Event } \\
\text { rate }\end{array}$ & $\begin{array}{c}\text { Lower } \\
\text { limit }\end{array}$ & $\begin{array}{c}\text { Upper } \\
\text { limit }\end{array}$ & Z-Value & $\mathrm{p}$-Value & & \\
\hline Ali-Mucheru 2016 & 0.001 & 0.000 & 0,022 & -4.632 & 0.000 & + & \\
\hline Ambicka 2016 & 0.006 & 0.000 & 0.089 & -3.600 & 0.000 & & \\
\hline Badr 2014 & 0.007 & 0.000 & 0.097 & -3.536 & 0.000 & & \\
\hline Barra 2017 & 0.042 & 0.003 & 0.425 & -2.170 & 0.030 & & \\
\hline Barra 2018 & 0.015 & 0.001 & 0.196 & -2.951 & 0.003 & & \\
\hline Bhimani 2017 & 0.000 & 0.000 & 0.003 & -5.964 & 0.000 & & \\
\hline Bicchierai 2018 & 0.012 & 0.001 & 0.167 & -3.088 & 0.002 & & \\
\hline Blum 2014 & 0.024 & 0.001 & 0.287 & -2.594 & 0.009 & & \\
\hline Brandan 2016 & 0.026 & 0.002 & 0.310 & -2.519 & 0.012 & & \\
\hline Cheung 2014 & 0.006 & 0.000 & 0.083 & -3.658 & 0.000 & 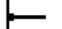 & \\
\hline Cheung 2016a & 0.002 & 0.000 & 0.030 & -4.408 & 0.000 & F & \\
\hline Cheung $2016 \mathrm{~b}$ & 0.006 & 0.000 & 0.084 & -3.642 & 0.000 & & \\
\hline Chou 2015 & 0.032 & 0.015 & 0.070 & -8.182 & 0.000 & 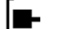 & \\
\hline Danala 2018 & 0.004 & 0.000 & 0.067 & -3.815 & 0.000 & & \\
\hline Deng 2018 & 0.004 & 0.000 & 0.054 & -3.985 & 0.000 & & \\
\hline Diekmann 2005 & 0.023 & 0.001 & 0.277 & -2.629 & 0.009 & & \\
\hline Diekmann 2011 & 0.014 & 0.002 & 0.094 & -4.204 & 0.000 & & \\
\hline Dromain 2006 & 0.024 & 0.001 & 0.287 & -2.594 & 0.009 & & \\
\hline Dromain 2011 & 0.004 & 0.000 & 0.063 & -3.870 & 0.000 & & \\
\hline Dromain 2012 & 0.009 & 0.001 & 0.062 & -4.670 & 0.000 & & \\
\hline Elsaid 2015 & 0.014 & 0.001 & 0.191 & -2.973 & 0.003 & & \\
\hline Fallenberg 2014a & 0.008 & 0.001 & 0.058 & -4.742 & 0.000 & & \\
\hline Fallenberg $2014 \mathrm{~b}$ & 0.006 & 0.000 & 0.091 & -3.582 & 0.000 & & \\
\hline $\begin{array}{l}\text { Fallenberg } 2017 \\
\text { F }\end{array}$ & 0.006 & 0.001 & 0.044 & -5.021 & 0.000 & & \\
\hline Francescone 2014 & $\begin{array}{l}4.0006 \\
4\end{array}$ & 0.000 & 0.083 & $\begin{array}{l}-3.650 \\
-3.050\end{array}$ & 0.000 & F & \\
\hline Gluskin 2017 & 0.083 & 0.005 & 0.622 & -1.623 & 0.105 & L & \\
\hline Helal 2017a & 0.005 & 0.000 & 0.076 & -3.726 & 0.000 & — & \\
\hline Helal 2017b & 0.016 & 0.001 & 0.211 & -2.883 & 0.004 & & \\
\hline Helal 2018 & 0.002 & 0.000 & 0.026 & -4.521 & 0.000 & - & \\
\hline Hill 2013 & 0.005 & 0.000 & 0.076 & -3.726 & 0.000 & 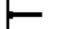 & \\
\hline Hobbs 2015 & 0.041 & 0.010 & 0.149 & -4.373 & 0.000 & & \\
\hline Houben 2017 & 0.006 & 0.002 & 0.014 & -11.407 & 0.000 & & \\
\hline Houben 2019 & 0.003 & 0.000 & 0.052 & -4.015 & 0.000 & F & \\
\hline Iotti 2017 & 0.009 & 0.001 & 0.129 & -3.302 & 0.001 & & \\
\hline James 2017 & 0.003 & 0.000 & 0.044 & -4.130 & 0.000 & E & \\
\hline Jeukens 2014 & 0.010 & 0.001 & 0.146 & -3.203 & 0.001 & & \\
\hline Jochelson 2013 & 0.006 & 0.000 & 0.089 & -3.600 & 0.000 & & \\
\hline Jochelson 2017 & 0.013 & 0.005 & 0.034 & -8.612 & 0.000 & $\mathbf{F}$ & \\
\hline Jong 2003 & 0.022 & 0.001 & 0.268 & -2.662 & 0.008 & & \\
\hline Kamal 2015 & 0.003 & 0.000 & 0.045 & -4.109 & 0.000 & - & \\
\hline Kamal 2016 & 0.002 & 0.000 & 0.032 & -4.360 & 0.000 & & \\
\hline Kariyappa 2016 & 0.011 & 0.001 & 0.154 & -3.156 & 0.002 & & \\
\hline Kim 2018 & 0.012 & 0.002 & 0.080 & -4.392 & 0.000 & & \\
\hline Klang 2018 & 0.001 & 0.000 & 0.008 & -5.340 & 0.000 & & \\
\hline Knogler 2016 & 0.031 & 0.002 & 0.350 & -2.390 & 0.017 & & \\
\hline Knogler 2017 & 0.042 & 0.003 & 0.425 & -2.170 & 0.030 & & \\
\hline Lalji 2016 & 0.003 & 0.000 & 0.039 & -4.230 & 0.000 & - & \\
\hline Lee-Felker 2017 & 0.009 & 0.001 & 0.134 & -3.275 & 0.001 & & \\
\hline Lewin 2003 & 0.019 & 0.001 & 0.236 & -2.781 & 0.005 & & \\
\hline Lewis 2017 & 0.002 & 0.000 & 0.037 & -4.261 & 0.000 & $F$ & \\
\hline Li 2017 & 0.010 & 0.001 & 0.143 & -3.218 & 0.001 & & \\
\hline Lobbes 2014 & 0.004 & 0.000 & 0.066 & -3.828 & 0.000 & $\models$ & \\
\hline Lobbes 2015 & 0.006 & 0.000 & 0.084 & -3.642 & 0.000 & & \\
\hline Luczynska 2014 & 0.003 & 0.000 & 0.050 & -4.038 & 0.000 & - & \\
\hline Luczynska 2015a & 0.005 & 0.000 & 0.073 & -3.755 & 0.000 & - & \\
\hline Luczynska 2015b & 0.003 & 0.000 & 0.044 & -4.134 & 0.000 & $F$ & \\
\hline Luczynska 2016a & 0.003 & 0.000 & 0.040 & -4.208 & 0.000 & L & \\
\hline Luczynska 2016b & 0.004 & 0.000 & 0.065 & -3.846 & 0.000 & - & \\
\hline Luczynska 2018 & 0.006 & 0.000 & 0.089 & -3.600 & 0.000 & & \\
\hline Mokhtar 2014 & 0.008 & 0.001 & 0.118 & -3.377 & 0.001 & & \\
\hline Mori 2017 & 0.007 & 0.000 & 0.100 & -3.507 & 0.000 & & \\
\hline Moustafa 2018 & 0.003 & 0.000 & 0.048 & -4.075 & 0.000 & $F$ & \\
\hline Navarro 2018 & 0.001 & 0.000 & 0.017 & -4.831 & 0.000 & & \\
\hline Patel 2017a & 0.006 & 0.000 & 0.083 & -3.650 & 0.000 & & \\
\hline Patel $2017 \mathrm{~b}$ & 0.002 & 0.000 & 0.017 & -6.006 & 0.000 & & \\
\hline Patel 2018a & 0.008 & 0.000 & 0.110 & -3.434 & 0.001 & & \\
\hline Patel $2018 b$ & 0.010 & 0.001 & 0.138 & -3.247 & 0.001 & & \\
\hline Patel 2018c & 0.016 & 0.001 & 0.211 & -2.883 & 0.004 & & \\
\hline Phillips 2017 & 0.013 & 0.001 & 0.175 & -3.052 & 0.002 & & \\
\hline Phillips 2018 & 0.011 & 0.001 & 0.151 & -3.172 & 0.002 & & \\
\hline Richter 2017 & 0.004 & 0.000 & 0.064 & -3.858 & 0.000 & & \\
\hline Saraya 2017 & 0.014 & 0.001 & 0.191 & -2.973 & 0.003 & & \\
\hline Savaridas 2017 & 0.030 & 0.008 & 0.113 & -4.826 & 0.000 & & \\
\hline Sogani 2017 & 0.002 & 0.000 & 0.028 & -4.467 & 0.000 & & \\
\hline Sorin 2018 & 0.005 & 0.002 & 0.015 & -9.177 & 0.000 & & \\
\hline Tardivel 2016 & 0.003 & 0.000 & 0.039 & -4.215 & 0.000 & & \\
\hline Tennant 2016 & 0.010 & 0.001 & 0.068 & -4.562 & 0.0 & & \\
\hline Tohamey 2018 & 0.003 & 0.000 & 0.043 & -4.150 & 0.000 & F & \\
\hline Travieso-Aja 2014 & 0.004 & 0.000 & 0.056 & -3.959 & 0.000 & & \\
\hline Travieso-Aja 2018 & 0.003 & 0.000 & 0.048 & -4.066 & 0.000 & $\leftarrow$ & \\
\hline Tsigginou 2016 & 0.005 & 0.001 & 0.032 & -5.358 & 0.000 & & \\
\hline Wang 2016 & 0.007 & 0.000 & 0.105 & -3.466 & 0.001 & & \\
\hline Xing 2018 & 0.002 & 0.000 & 0.033 & -4.348 & 0.000 & - & \\
\hline Yagil 2016 & 0.002 & 0.000 & 0.038 & -4.233 & 0.000 & - & \\
\hline & 0.008 & 0.006 & 0.010 & -38.400 & 0.000 & & \\
\hline
\end{tabular}

Fig. 5 Forest plot of the 84 analysed articles on contrast-enhanced spectral mammography. No heterogeneity was found among studies $\left(P^{R}=0 \%\right)$. The last row shows the pooled rate for adverse reactions arising from iodinated contrast agent administration, calculated using the fixed-effect model 


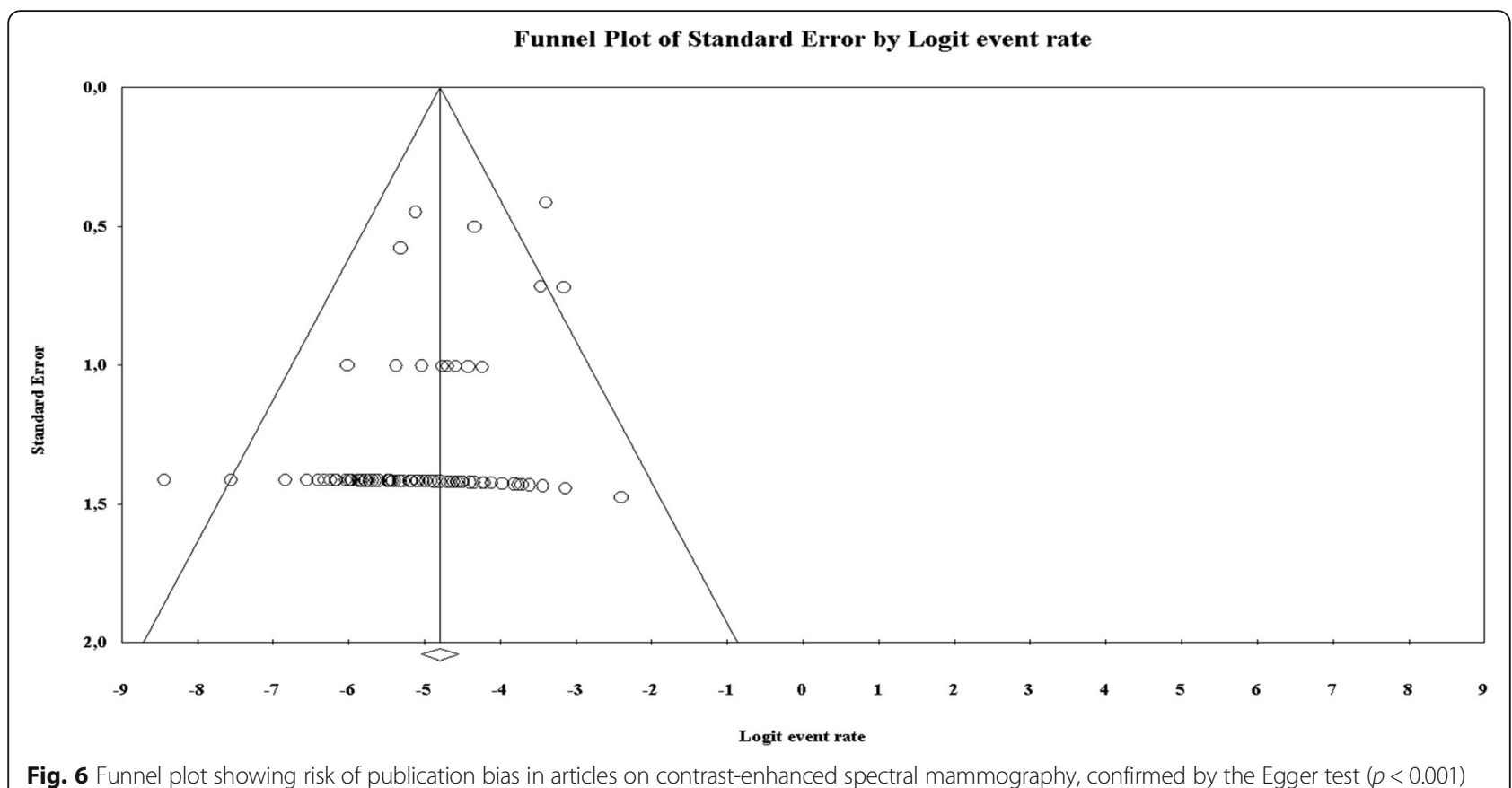

Fig. 6 Funnel plot showing risk of publication bias in articles on contrast-enhanced spectral mammography, confirmed by the Egger test $(p<0.001)$

AGD increase, studies specifically devised to ascertain CESM effective AGD found a substantial AGD increment ranging 42-80\% [56, 60, 82]. While CESM AGDs remain under the threshold stated by European guidelines for screening mammography [112], further studies are needed to investigate CESM AGD [56, 82].

Furthermore, we remark the absence of standardised protocols. This methodological void, especially regarding the acquisition workflow, represents a threat to reproducibility and comparison of imaging results. While $98 \%$ of all studies reporting the total examination time completed the examination before $10 \mathrm{~min}$ from contrast administration, and while some studies presented evidence on the irrelevance of the acquisition order [55, 64], there are no studies comparing different approaches.

The pooled rate of adverse reactions to ICA administration was $0.82 \%(0.64-1.05 \% 95 \% \mathrm{CI})$ with a total of 30 adverse reactions in 14012 patients, a rate similar to that reported for CT $0.6 \%$ [113] in 84928 adult patients or $0.7 \%$ [114] in 29508 patients (given Iopromide, which is also used for CESM). Particularly, considering only severe adverse reactions in CT, Wang et al. [113] reported $11 / 84928(0.0129 \%)$ reactions, as well as Mortelé et al. [114] 4/29508 (0.0135\%). These rates seem to be higher than that found in our meta-analysis $1 / 14012(0.007 \%)$, a comparison to consider with caution due to the nature

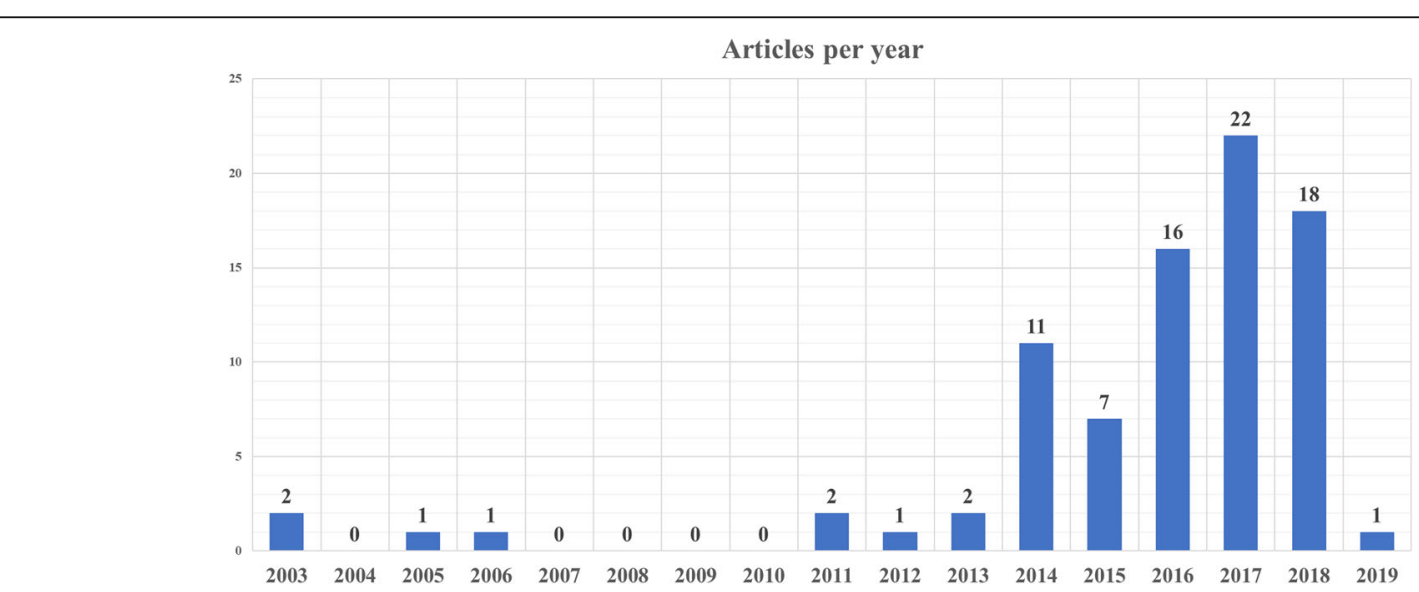

Fig. 7 Graphic showing the number of articles published per year regarding contrast-enhanced spectral mammography 
of rare events such as severe reactions to ICA. One aspect to consider is the different profile of patients undergoing CESM compared to those requiring contrastenhanced CT, the former being that of basically "healthy" subjects, the latter implying the possibility of relevant disease, including also serious emergency conditions.

This review has limitations. Patient data are probably shared and duplicate among some studies from the same research group. This has been shown to negatively impact on review quality $[115,116]$ and could only be prevented via individual patient data sharing [117]. However, for technical aspects of this systematic review, our choice to evaluate study groups rather than single articles should have mitigated this bias. Conversely, our pooled rate of adverse reactions could be underestimated.

In conclusion, our review shows that CESM is unevenly performed across different centres, in terms of contrast agent type and concentration and order of view acquisition. However, most research groups performed CESM using a contrast dose of $1.5 \mathrm{~mL} / \mathrm{kg}$, factory-set $\mathrm{kVp}$ ranges for low- and high-energy acquisitions, beginning image acquisition after $120 \mathrm{~s}$ from contrast agent injection and completing the examination within $10 \mathrm{~min}$. Further studies are needed to investigate the role of background parenchymal enhancement and to harvest data that can firmly back up subsequent technical guidelines and consensus statements for standardised CESM protocols.

\section{Abbreviations}

AGD: Average glandular dose; CC: Craniocaudal; CESM: Contrast-enhanced spectral mammography; Cl: Confidence interval; CT: Computed tomography; DM: Digital mammography; ICA: lodinated contrast agent; kVp: Peak kilovoltage; MLO: Mediolateral oblique; MRI: Magnetic resonance imaging; PRISMA: Preferred Reporting Items for Systematic Reviews and Meta-Analyses

\section{Authors' contributions}

Each author has participated sufficiently in this work to take public responsibility for its content. The manuscript is approved by all authors and by the responsible authorities. All authors read and approved the final manuscript.

\section{Funding}

This research did not receive any specific grant from funding agencies in the public, commercial or not-for-profit sectors.

\section{Availability of data and materials}

The datasets used and analysed during the current study are available from the corresponding author on reasonable request.

\section{Ethics approval and consent to participate}

Not applicable

\section{Consent for publication}

Not applicable

\section{Competing interests}

FS declares to have received grants from or to be member of speakers' bureau/advisory board for Bayer, Bracco, and General Electric. All other authors declare that they have no competing interests.

\section{Author details}

'Department of Biomedical Sciences for Health, Università degli Studi di Milano, Via Mangiagalli 31, 20133 Milan, Italy. ${ }^{2}$ Department of Morphology, Surgery and Experimental Medicine, Section of Radiology, University of Ferrara, Via Ludovico Ariosto 35, 44121 Ferrara, Italy. ${ }^{3}$ Unit of Radiology, IRCCS Policlinico San Donato, Via Morandi 30, 20097 San Donato Milanese, Italy.

Received: 25 March 2019 Accepted: 17 May 2019

Published online: 02 August 2019

\section{References}

1. Frigerio A, Sardanelli F, Podo F (2017) Radiological screening of breast cancer: evolution. In: Veronesi U, Goldhirsch A, Veronesi P, Gentilini OD, Leonardi MC (eds) Breast Cancer. Springer International Publishing, Cham, pp 171-203

2. Pisano ED, Gatsonis C, Hendrick E et al (2005) Diagnostic performance of digital versus film mammography for breast-cancer screening. N Engl J Med 353:1773-1783

3. Gilbert FJ, Tucker L, Young KC (2016) Digital breast tomosynthesis (DBT): a review of the evidence for use as a screening tool. Clin Radiol 71:141-150

4. Pattacini P, Nitrosi A, Giorgi Rossi P et al (2018) Digital mammography versus digital mammography plus tomosynthesis for breast cancer screening: the Reggio Emilia Tomosynthesis randomized trial. Radiology 288:375-385

5. Marinovich ML, Hunter KE, Macaskill P, Houssami N (2018) Breast cancer screening using tomosynthesis or mammography: a meta-analysis of cancer detection and recall. J Natl Cancer Inst 110:942-949

6. Patel BK, Lobbes MBI, Lewin J (2018) Contrast enhanced spectral mammography: a review. Semin Ultrasound CT MRI 39:70-79

7. Jong RA, Yaffe MJ, Skarpathiotakis M et al (2003) Contrast-enhanced digital mammography: initial clinical experience. Radiology 228:842-850

8. Diekmann F, Diekmann S, Jeunehomme F, Muller S, Hamm B, Bick U (2005) Digital mammography using iodine-based contrast media. Invest Radiol 40: 397-404

9. Dromain C, Balleyguier C, Muller S et al (2006) Evaluation of tumor angiogenesis of breast carcinoma using contrast-enhanced digital mammography. AJR Am J Roentgenol 187:W528-W537

10. Hill ML, Mainprize JG, Carton A-K et al (2013) Anatomical noise in contrastenhanced digital mammography. Part II. Dual-energy imaging. Med Phys 40: 081907

11. Dromain C, Balleyguier C, Adler G, Garbay JR, Delaloge S (2009) Contrastenhanced digital mammography. Eur J Radiol 69:34-42

12. Skarpathiotakis M, Yaffe MJ, Bloomquist AK et al (2002) Development of contrast digital mammography. Med Phys 29:2419-2426

13. Bhimani C, Matta D, Roth RG et al (2017) Contrast-enhanced spectral mammography. Acad Radiol 24:84-88

14. Chou C-P, Lewin JM, Chiang C-L et al (2015) Clinical evaluation of contrastenhanced digital mammography and contrast enhanced tomosynthesis-comparison to contrast-enhanced breast MRI. Eur J Radiol 84:2501-2508

15. Tennant SL, James JJ, Cornford EJ et al (2016) Contrast-enhanced spectral mammography improves diagnostic accuracy in the symptomatic setting. Clin Radiol 71:1148-1155

16. Lewis TC, Pizzitola VJ, Giurescu ME et al (2017) Contrast-enhanced digital mammography: a single-institution experience of the first 208 cases. Breast J 23:67-76

17. Moustafa AFI, Kamal EF, Hassan MM, Sakr M, Gomaa MMM (2018) The added value of contrast enhanced spectral mammography in identification of multiplicity of suspicious lesions in dense breast. Egypt J Radiol Nucl Med 49:259-264

18. Saraya S, Adel L, Mahmoud A (2017) Indeterminate breast lesions: can contrast enhanced digital mammography change our decisions? Egypt J Radiol Nucl Med 48:547-552

19. Tardivel A-M, Balleyguier C, Dunant A et al (2016) Added value of contrastenhanced spectral mammography in postscreening assessment. Breast J 22: $520-528$

20. Lobbes MB, Lalji U, Houwers J et al (2014) Contrast-enhanced spectral mammography in patients referred from the breast cancer screening programme. Eur Radiol 24:1668-1676 
21. Lalji UC, Houben IP, Prevos R et al (2016) Contrast-enhanced spectral mammography in recalls from the Dutch breast cancer screening program: validation of results in a large multireader, multicase study. Eur Radiol 26: 4371-4379

22. Houben IP, Vanwetswinkel S, Kalia V et al (2019) Contrast-enhanced spectral mammography in the evaluation of breast suspicious calcifications: diagnostic accuracy and impact on surgical management. Acta Radiol. [Epub ahead of print]

23. Patel BK, Davis J, Ferraro C et al (2018) Value added of preoperative contrast-enhanced digital mammography in patients with invasive lobular carcinoma of the breast. Clin Breast Cancer 18:e1339-e1345

24. Travieso-Aja MDM, Naranjo-Santana P, Fernández-Ruiz C et al (2018) Factors affecting the precision of lesion sizing with contrast-enhanced spectral mammography. Clin Radiol 73:296-303

25. Helal MH, Mansour SM, Salaleldin LA, Alkalaawy BM, Salem DS, Mokhtar NM (2018) The impact of contrast-enhanced spectral mammogram (CESM) and three-dimensional breast ultrasound (3DUS) on the characterization of the disease extend in cancer patients. Br J Radiol 91:20170977

26. Lee-Felker SA, Tekchandani L, Thomas M et al (2017) Newly diagnosed breast cancer: comparison of contrast-enhanced spectral mammography and breast MR imaging in the evaluation of extent of disease. Radiology 285:389-400

27. Patel BK, Garza SA, Eversman S, Lopez-Alvarez Y, Kosiorek H, Pockaj BA (2017) Assessing tumor extent on contrast-enhanced spectral mammography versus full-field digital mammography and ultrasound. Clin Imaging 46:78-84

28. Helal MH, Mansour SM, Zaglol M, Salaleldin LA, Nada OM, Haggag MA (2017) Staging of breast cancer and the advanced applications of digital mammogram: what the physician needs to know? Br J Radiol 90:20160717

29. Ambicka A, Luczynska E, Adamczyk A, Harazin-Lechowska A, Sas-Korczynska B, Niemiec J (2016) The tumour border on contrast-enhanced spectral mammography and its relation to histological characteristics of invasive breast cancer. Pol J Pathol 3:295-299

30. Lobbes MB, Lalji UC, Nelemans PJ et al (2015) The quality of tumor size assessment by contrast-enhanced spectral mammography and the benefit of additional breast MRI. J Cancer 6:144-150

31. Blum KS, Rubbert C, Mathys B, Antoch G, Mohrmann S, Obenauer S (2014) Use of contrast-enhanced spectral mammography for intramammary cancer staging. Acad Radiol 21:1363-1369

32. Fallenberg EM, Dromain C, Diekmann F et al (2014) Contrast-enhanced spectral mammography versus MRI: initial results in the detection of breast cancer and assessment of tumour size. Eur Radiol 24:256-264

33. Ali-Mucheru M, Pockaj B, Patel B et al (2016) Contrast-enhanced digital mammography in the surgical management of breast cancer. Ann Surg Oncol 23:649-655

34. Patel BK, Ranjbar S, Wu T et al (2018) Computer-aided diagnosis of contrast-enhanced spectral mammography: a feasibility study. Eur J Radiol 98:207-213

35. Luczyńska E, Heinze-Paluchowska S, Dyczek S, Blecharz P, Rys J, Reinfuss M (2014) Contrast-enhanced spectral mammography: comparison with conventional mammography and histopathology in 152 women. Korean J Radiol 15:689

36. Luczynska E, Niemiec J, Heinze S et al (2018) Intensity and pattern of enhancement on CESM: prognostic significance and its relation to expression of podoplanin in tumor stroma - a preliminary report. Anticancer Res 38:1085-1095

37. Iotti V, Ravaioli S, Vacondio R et al (2017) Contrast-enhanced spectral mammography in neoadjuvant chemotherapy monitoring: a comparison with breast magnetic resonance imaging. Breast Cancer Res 19:106

38. Patel BK, Hilal T, Covington M et al (2018) Contrast-enhanced spectral mammography is comparable to MRI in the assessment of residual breast cancer following neoadjuvant systemic therapy. Ann Surg Oncol 25:13501356

39. Barra FR, de Souza FF, Camelo REFA, Ribeiro ACO, Farage L (2017) Accuracy of contrast-enhanced spectral mammography for estimating residual tumor size after neoadjuvant chemotherapy in patients with breast cancer: a feasibility study. Radiol Bras 50:224-230

40. Barra FR, Sobrinho AB, Barra RR et al (2018) Contrast-enhanced mammography (CEM) for detecting residual disease after neoadjuvant chemotherapy: a comparison with breast magnetic resonance imaging (MRI). Biomed Res Int 2018:1-9
41. Tagliafico AS, Bignotti B, Rossi F et al (2016) Diagnostic performance of contrast-enhanced spectral mammography: systematic review and metaanalysis. Breast 28:13-19

42. Zhu X, Huang J-M, Zhang K et al (2018) Diagnostic value of contrastenhanced spectral mammography for screening breast cancer: systematic review and meta-analysis. Clin Breast Cancer 18:e985-e995

43. Sardanelli F, Boetes C, Borisch B et al (2010) Magnetic resonance imaging of the breast: recommendations from the EUSOMA working group. Eur J Cancer 46:1296-1316

44. Mann RM, Kuhl CK, Kinkel K, Boetes C (2008) Breast MRl: guidelines from the European Society of Breast Imaging. Eur Radiol 18:1307-1318

45. The American Society of Breast Surgeons. Consensus guideline on diagnostic and screening magnetic resonance imaging of the breast. https://www. breastsurgeons.org/about/statements/PDF_Statements/MRI.pdf. Accessed 30 May 2019.

46. American College of Radiology. ACR practice parameter for the performance of contrast-enhanced magnetic resonance imaging (MRI) of the breast. Available from: https://www.acr.org/-/media/ACR/Files/PracticeParameters/mr-contrast-breast.pdf. Accessed 30 May 2019.

47. Zanardo M, Cozzi A, Trimboli RM, Carbonaro LA, Sardanelli F. Technique and diagnostic performance of contrast-enhanced spectral mammography: a systematic review. PROSPERO 2018 CRD42018118554. Available from: https://www.crd.york.ac.uk/PROSPERO/display_record.php?ID= CRD42018118554. Accessed 30 May 2019.

48. Moher D, Liberati A, Tetzlaff J, Altman DG (2009) Preferred Reporting Items for Systematic Reviews and Meta-Analyses: the PRISMA statement. PLoS Med 6:e1000097

49. Egger M, Davey Smith G, Schneider M, Minder C (1997) Bias in meta-analysis detected by a simple, graphical test. BMJ 315:629-634

50. Luczyńska E, Heinze S, Adamczyk A, Rys J, Mitus JW, Hendrick E (2016) Comparison of the mammography, contrast-enhanced spectral mammography and ultrasonography in a group of 116 patients. Anticancer Res 36:4359-4366

51. Tohamey YM, Youssry SW, Abd El Aziz Al (2018) Interpretation of patterns of enhancement on contrast-enhanced spectral mammography: an approach to a standardized scheme. Egypt J Radiol Nucl Med 49:854-868

52. Dromain C, Thibault F, Diekmann F et al (2012) Dual-energy contrastenhanced digital mammography: initial clinical results of a multireader, multicase study. Breast Cancer Res 14:R94

53. Łuczyńska E, Heinze-Paluchowska S, Hendrick E et al (2015) Comparison between breast MRI and contrast-enhanced spectral mammography. Med Sci Monit 21:1358-1367

54. Jochelson MS, Pinker K, Dershaw DD et al (2017) Comparison of screening CEDM and MRI for women at increased risk for breast cancer: a pilot study. Eur J Radiol 97:37-43

55. Jochelson MS, Dershaw DD, Sung JS et al (2013) Bilateral contrast-enhanced dual-energy digital mammography: feasibility and comparison with conventional digital mammography and MR imaging in women with known breast carcinoma. Radiology 266:743-751

56. James JR, Pavlicek W, Hanson JA, Boltz TF, Patel BK (2017) Breast radiation dose with CESM compared with 2D FFDM and 3D tomosynthesis mammography. AJR Am J Roentgenol 208:362-372

57. Mokhtar O, Mahmoud S (2014) Can contrast enhanced mammography solve the problem of dense breast lesions? Egypt J Radiol Nucl Med 45: 1043-1052

58. Houben IPL, Van de Voorde P, Jeukens CRLPN et al (2017) Contrast-enhanced spectral mammography as work-up tool in patients recalled from breast cancer screening has low risks and might hold clinical benefits. Eur J Radiol 94:31-37

59. Dromain C, Thibault F, Muller S et al (2011) Dual-energy contrast-enhanced digital mammography: initial clinical results. Eur Radiol 21:565-574

60. Jeukens CRLPN, Lalji UC, Meijer E et al (2014) Radiation exposure of contrast-enhanced spectral mammography compared with full-field digital mammography. Invest Radiol 49:659-665

61. Diekmann F, Freyer M, Diekmann S et al (2011) Evaluation of contrastenhanced digital mammography. Eur J Radiol 78:112-121

62. Richter V, Hatterman V, Preibsch H et al (2018) Contrast-enhanced spectral mammography in patients with MRI contraindications. Acta Radiol 59:798-805

63. Gluskin J, Click M, Fleischman R, Dromain C, Morris EA, Jochelson MS (2017) Contamination artifact that mimics in-situ carcinoma on contrast-enhanced digital mammography. Eur J Radiol 95:147-154 
64. Travieso Aja MM, Rodríguez Rodríguez M, Alayón Hernández S, Vega Benítez V, Luzardo OP (2014) Dual-energy contrast-enhanced mammography. Radiologia 56:390-399

65. Patel BK, Naylor ME, Kosiorek HE et al (2017) Clinical utility of contrastenhanced spectral mammography as an adjunct for tomosynthesisdetected architectural distortion. Clin Imaging 46:44-52

66. Francescone MA, Jochelson MS, Dershaw DD et al (2014) Low energy mammogram obtained in contrast-enhanced digital mammography (CEDM) is comparable to routine full-field digital mammography (FFDM). Eur J Radiol 83:1350-1355

67. Łuczyńska E, Niemiec J, Hendrick E et al (2016) Degree of enhancement on contrast enhanced spectral mammography (CESM) and lesion type on mammography (MG): comparison based on histological results. Med Sci Monit 22:3886-3893

68. Kariyappa KD, Gnanaprakasam F, Anand S, Krishnaswami M, Ramachandran M (2016) Contrast enhanced dual energy spectral mammogram, an emerging addendum in breast imaging. Br J Radiol 89:20150609

69. Danala G, Patel B, Aghaei F et al (2018) Classification of breast masses using a computer-aided diagnosis scheme of contrast enhanced digital mammograms. Ann Biomed Eng 46:1419-1431

70. Phillips J, Miller MM, Mehta TS et al (2017) Contrast-enhanced spectral mammography (CESM) versus MRI in the high-risk screening setting: patient preferences and attitudes. Clin Imaging 42:193-197

71. Yagil Y, Shalmon A, Rundstein A et al (2016) Challenges in contrastenhanced spectral mammography interpretation: artefacts lexicon. Clin Radiol 71:450-457

72. Cheung Y-C, Tsai H-P, Lo Y-F, Ueng S-H, Huang P-C, Chen S-C (2016) Clinical utility of dual-energy contrast-enhanced spectral mammography for breast microcalcifications without associated mass: a preliminary analysis. Eur Radiol 26:1082-1089

73. ElSaid NAE, Farouk S, Shetat OMM, Khalifa NM, Nada OM (2015) Contrast enhanced digital mammography: is it useful in detecting lesions in edematous breast? Egypt J Radiol Nucl Med 46:811-819

74. Mori M, Akashi-Tanaka S, Suzuki S et al (2017) Diagnostic accuracy of contrast-enhanced spectral mammography in comparison to conventional full-field digital mammography in a population of women with dense breasts. Breast Cancer 24:104-110

75. Savaridas SL, Taylor DB, Gunawardana D, Phillips M (2017) Could parenchymal enhancement on contrast-enhanced spectral mammography (CESM) represent a new breast cancer risk factor? Correlation with known radiology risk factors. Clin Radiol 72:1085.e1-1085.e9

76. Fallenberg EM, Schmitzberger FF, Amer H et al (2017) Contrast-enhanced spectral mammography vs. mammography and MRI - clinical performance in a multi-reader evaluation. Eur Radiol 27:2752-2764

77. Brandan M-E, Cruz-Bastida JP, Rosado-Méndez IM et al (2016) Clinical study of contrast-enhanced digital mammography and the evaluation of blood and lymphatic microvessel density. Br J Radiol 89:20160232

78. Deng C-Y, Juan Y-H, Cheung Y-C et al (2018) Quantitative analysis of enhanced malignant and benign lesions on contrast-enhanced spectral mammography. Br J Radiol 91:20170605

79. Mohamed Kamal R, Hussien Helal M, Wessam R, Mahmoud Mansour S, Godda I, Alieldin N (2015) Contrast-enhanced spectral mammography: impact of the qualitative morphology descriptors on the diagnosis of breast lesions. Eur J Radiol 84:1049-1055

80. Sogani J, Morris EA, Kaplan JB et al (2017) Comparison of background parenchymal enhancement at contrast-enhanced spectral mammography and breast MR imaging. Radiology 282:63-73

81. Hobbs MM, Taylor DB, Buzynski S, Peake RE (2015) Contrast-enhanced spectral mammography (CESM) and contrast enhanced MRI (CEMRI): patient preferences and tolerance. J Med Imaging Radiat Oncol 59:300-305

82. Phillips J, Mihai G, Hassonjee SE et al (2018) Comparative dose of contrastenhanced spectral mammography (CESM), digital mammography, and digital breast tomosynthesis. AJR Am J Roentgenol 211:839-846

83. Knogler T, Homolka P, Hörnig M et al (2016) Contrast-enhanced dual energy mammography with a novel anode/filter combination and artifact reduction: a feasibility study. Eur Radiol 26:1575-1581

84. Xing D, Lv Y, Sun B et al (2018) Diagnostic value of contrast-enhanced spectral mammography in comparison to magnetic resonance imaging in breast lesions. J Comput Assist Tomogr 43:245-251

85. Fallenberg EM, Dromain C, Diekmann F et al (2014) Contrast-enhanced spectral mammography: does mammography provide additional clinical benefits or can some radiation exposure be avoided? Breast Cancer Res Treat 146:371-381

86. Cheung Y-C, Lin Y-C, Wan Y-L et al (2014) Diagnostic performance of dualenergy contrast-enhanced subtracted mammography in dense breasts compared to mammography alone: interobserver blind-reading analysis. Eur Radiol 24:2394-2403

87. Kim EY, Youn I, Lee KH et al (2018) Diagnostic value of contrast-enhanced digital mammography versus contrast-enhanced magnetic resonance imaging for the preoperative evaluation of breast cancer. J Breast Cancer 21:453

88. Klang E, Krosser A, Amitai MM et al (2018) Utility of routine use of breast ultrasound following contrast-enhanced spectral mammography. Clin Radiol 73:908.e11-908.e16

89. Tsigginou A, Gkali C, Chalazonitis A et al (2016) Adding the power of iodinated contrast media to the credibility of mammography in breast cancer diagnosis. Br J Radiol 89:20160397

90. Navarro ME, Razmilic D, Araos I, Rodrigo A, Andia ME (2018) Contrastenhanced spectral mammography. Experience in 465 examinations. Rev Med Chil 146:141-149

91. Luczynska E, Niemiec J, Ambicka A et al (2015) Correlation between blood and lymphatic vessel density and results of contrast-enhanced spectral mammography. Pol J Pathol 3:310-322

92. Sorin V, Yagil Y, Yosepovich A et al (2018) Contrast-enhanced spectral mammography in women with intermediate breast cancer risk and dense breasts. AJR Am J Roentgenol 211:W267-W274

93. Bicchierai G, Nori J, De Benedetto D et al (2018) Role of contrast-enhanced spectral mammography in the post biopsy management of B3 lesions: preliminary results. Tumori J. [Epub ahead of print]

94. Knogler T, Homolka P, Hoernig M et al (2017) Application of BI-RADS descriptors in contrast-enhanced dual-energy mammography: comparison with MRI. Breast Care (Basel) 12:212-216

95. Kamal RM, Helal MH, Mansour SM et al (2016) Can we apply the MRI BIRADS lexicon morphology descriptors on contrast-enhanced spectral mammography? Br J Radiol 89:20160157

96. Lewin JM, Isaacs PK, Vance V, Larke FJ (2003) Dual-energy contrast-enhanced digital subtraction mammography: feasibility. Radiology 229:261-268

97. Wang Q, Li K, Wang L, Zhang J, Zhou Z, Feng Y (2016) Preclinical study of diagnostic performances of contrast-enhanced spectral mammography versus MRI for breast diseases in China. Springerplus 5:763

98. Cheung Y-C, Juan Y-H, Lin Y-C et al (2016) Dual-energy contrast-enhanced spectral mammography: enhancement analysis on BI-RADS 4 non-mass microcalcifications in screened women. PLoS One 11:e0162740

99. Helal M, Abu Samra MF, Ibraheem MA, Salama A, Hassan EE, Hassan NE-H (2017) Accuracy of CESM versus conventional mammography and ultrasound in evaluation of BI-RADS 3 and 4 breast lesions with pathological correlation. Egypt J Radiol Nucl Med 48:741-750

100. Li L, Roth R, Germaine P et al (2017) Contrast-enhanced spectral mammography (CESM) versus breast magnetic resonance imaging (MRI): a retrospective comparison in 66 breast lesions. Diagn Interv Imaging 98:113-123

101. Badr S, Laurent N, Régis C, Boulanger L, Lemaille S, Poncelet E (2014) Dualenergy contrast-enhanced digital mammography in routine clinical practice in 2013. Diagn Interv Imaging 95:245-258

102. Covington MF, Pizzitola VJ, Lorans R et al (2018) The future of contrastenhanced mammography. AJR Am J Roentgenol 210:292-300

103. Lancaster RB, Gulla S, De Los Santos J, Umphrey HR (2018) Contrast-enhanced spectral mammography in breast imaging. Semin Roentgenol 53:294-300

104. James JJ, Tennant SL (2018) Contrast-enhanced spectral mammography (CESM). Clin Radiol 73:715-723

105. Patel BK, Gray RJ, Pockaj BA (2017) Potential cost Savings of ContrastEnhanced Digital Mammography. AJR Am J Roentgenol 208:W231-W237

106. Lewin J (2018) Comparison of contrast-enhanced mammography and contrastenhanced breast MR imaging. Magn Reson Imaging Clin N Am 26:259-263

107. Minsinger KD, Kassis HM, Block CA, Sidhu M, Brown JR (2014) Meta-analysis of the effect of automated contrast injection devices versus manual injection and contrast volume on risk of contrast-induced nephropathy. Am J Cardiol 113:49-53

108. Endrikat J, Barbati R, Scarpa M, Jost G, Ned Uber AE 3rd (2018) Accuracy and repeatability of automated injector versus manual administration of an MRI contrast agent—results of a laboratory study. Invest Radiol 53:1-5

109. Jost G, Endrikat J, Pietsch H (2017) The impact of injector-based contrast agent administration on bolus shape and magnetic resonance angiography image quality. Magn Reson Insights 10:1178623X1770589 
110. Auler MA, Heagy T, Aganovic L, Brothers R, Costello P, Schoepf UJ (2006) Saline chasing technique with dual-syringe injector systems for multidetector row computed tomographic angiography: rationale, indications, and protocols. Curr Probl Diagn Radiol 35:1-11

111. Kidoh M, Nakaura T, Awai K et al (2013) Novel connecting tube for saline chaser in contrast-enhanced CT: the effect of spiral flow of saline on contrast enhancement. Eur Radiol 23:3213-3218

112. Perry N, Broeders M, de Wolf C, Tornberg S, Holland R, von Karsa L (2007) European guidelines for quality assurance in breast cancer screening and diagnosis. Fourth edition--summary document. Ann Oncol 19:614-622

113. Wang CL, Cohan RH, Ellis JH, Caoili EM, Wang G, Francis IR (2008) Frequency, outcome, and appropriateness of treatment of nonionic iodinated contrast media reactions. AJR Am J Roentgenol 191:409-415

114. Mortelé KJ, Oliva M-R, Ondategui S, Ros PR, Silverman SG (2005) Universal use of nonionic iodinated contrast medium for CT: evaluation of safety in a large urban teaching hospital. AJR Am J Roentgenol 184:31-34

115. Huston P, Moher D (1996) Redundancy, disaggregation, and the integrity of medical research. Lancet 347:1024-1026

116. Murphy L, Wyllie A (2009) Duplicate patient data in a meta-analysis: a threat to validity. J Crit Care 24:466-467

117. Sardanelli F, Alì M, Hunink MG, Houssami N, Sconfienza LM, Di Leo G (2018) To share or not to share? Expected pros and cons of data sharing in radiological research. Eur Radiol 28:2328-2335

\section{Publisher's Note}

Springer Nature remains neutral with regard to jurisdictional claims in published maps and institutional affiliations.

\section{Submit your manuscript to a SpringerOpen ${ }^{\circ}$ journal and benefit from:}

- Convenient online submission

- Rigorous peer review

- Open access: articles freely available online

High visibility within the field

- Retaining the copyright to your article

Submit your next manuscript at $\boldsymbol{\nabla}$ springeropen.com 